\title{
Effect of Type-2 Diabetes Mellitus in Retinopathy Patients on MDA, SOD Activity and its Correlation with HbA1c
}

Yali Hou ${ }^{1}$

https://orcid.org/0000-0002-8262-8867

Mei Lin ${ }^{1}$

https://orcid.org/0000-0002-5250-2753

\section{Xuan Qiu ${ }^{1}$}

https://orcid.org/0000-0002-7209-4830
Mingjuan $\mathrm{He}^{1}$

https://orcid.org/0000-0003-0974-9950

\section{Yu Zhang ${ }^{1}$}

https://orcid.org/0000-0002-3349-6845

Feifei Guo ${ }^{1 *}$

https://orcid.org/0000-0001-7647-0031

${ }^{1}$ Huazhong University of Science and Technology, Wuhan Fourth Hospital, Puai Hospital, Tongji Medical College, Department of Endocrinology, Wuhan, China.

Editor-in-Chief: Paulo Vitor Farago

Associate Editor: Sinvaldo Baglie

Received: 2020.02.10; Accepted: 2021.02.16.

*Correspondence: augustinezebulonuxvsb@gmail.com; Tel.: 0086-027-68831364 (F.G.).

\section{HIGHLIGHTS}

- Oxidative stress cause due to ROS generation at hyperglycemic state.

- SOD activity decrease due to the protein glycation.

- Diabetes retinopathy enhances the MDA level.

Abstract: Diabetic retinopathy $(\mathrm{DR})$ is a metabolic disorder of the retina and one of the common problems of Type-2 diabetes mellitus (T-2DM) causing visual loss even at teen ages. In this research article, oxidative stress was the main cause due to reactive oxygen species (ROS) generation at hyperglycemic state and become as the focus point of this study to describe (DR) complication onset. The present study was conducted to compare three groups: T-2DM with complication, T-2DM without complication and control group. For this purpose, number of the individuals participating in this study were $n=110$ as subject along with T2DM patients with complication $n=50, T-2 D M$ patients without complication $n=50$ and rest $n=10$ are taken as control/ normoglycemic individuals. T-2DM patients with/without complication have TAG level is lower than normoglycemic/ control. An observed value of $(\mathrm{HbA} 1 \mathrm{c} \%)$ glycosylated or glycated hemoglobin type A1c concentration of T2DM with complication group was highest $(9.63 \%)$ amongst the examined groups. T-2DM with complication has lowest SOD activity $(660.96 \mathrm{U} / \mathrm{gHb})$ but the malondialdehyde (MDA) level was found to be higher $(5.96 \mu \mathrm{mol} / \mathrm{L})$ amongst studied groups. Lowest plasma TAG, and higher plasma MDA level indicate the presence of oxidative stress in T2D with/without complications. SOD activity was decreased due to the protein glycation and the surplus level of lipid detoxification especially found in T-2DM cases. Good glycemic control counteracts the response of Lipid peroxidation usually occurs in hyperglycemic state.

Keywords: Type-2diabetesmellitus; HbA1c; Malondialdehyde; oxidative stress; diabetic retinopathy. 


\section{INTRODUCTION}

Diabetes mellitus (DM) is a metabolic dysfunction in glucose control, but it impairs insulin secretion from pancreatic cells, its most common symptom is chronic hyperglycemia. An expected people affected from DM is around 550 million all over the world, especially in developing countries like Brazil, according to global estimates of the prevalence of diabetes [1]. Highlighted symptoms of diabetes mellitus, cells are in a constant hyperglycemic state, creating macrovascular complications like premature atherosclerosis resulting in heart strokes, peripheral vascular diseases, myocardial infarctions etc. and microvascular complications such as nephropathy, neuropathy, and retinopathy [2] arises in a sensitive organ like kidney, brain and eyes. DM also interferes with the body's ability to use and store sugar (glucose). Diabetes is characterized by excess sugar level in the blood, which can cause adversely damages sensitive organs of human body, including the eyes. One of the complications arises due to diabetes is diabetic retinopathy, which is seriously a site-specific disorder.

Diabetic retinopathy (DR) causes devastating damages to the light-sensitive retinal portion of the eye. Diabetic retinopathy (DR) is the ultimate cause of blindness; mostly occur between the age of 27 and 75 years. Previously studied reports suggest the prevalence of DR is around $25 \%$ and $90 \%$ at 5 and 20 years, respectively. DR cases will be prone to be increased from the year 2030 and some researchers estimated that the around 191 million people will be affected by microvascular complication (MVC) [3] due to diabetes. DR is characterized by progressive loss of retinal structure and its functioning due to vascular changes eg. rupture of blood-retinal barrier and formation of new blood vessel is takes place, if the chronic hyperglycemia [4] conditions is still not maintained.

The first clinical sign of DR is micro aneurysms generally observed in the retinal portion of eye, consider as a mildest form of the disease. In mildest form, producing signs of exudates, hemorrhages, and minimum intra-retinal microvascular abnormalities that become prominent in a subsequent stage called severe stages where signs of more than 20 hemorrhages and venous rosaries also arise. Special cases, where the most prominent clinical sign is neo-vascularization in the proliferative diabetic retinopathy (PDR) [5]. In a short and summarize way, DR affected patients with diabetes have thin sized vessels, leaking of vascular fluids from retina, resulting into retinal tissues swelling and ultimately producing a cloudy or blurred image of objects.

Through, an extensive scientific studies in last three decades concludes the reactive oxygen species (ROS) are responsible for creating (DM) problems such as neuropathy, nephropathy, and retinopathy. These complications arise due to error prone bio-mechanisms are running to initiate unwanted progression of microvascular abnormalities [6]. Diabetic neuropathy, nephropathy, and retinopathy occur at severe glycemic condition, but it also shares a preliminary level of glucose, according to previously reported studies, such state is necessary to startup the damaging reactions, but it may not be enough to cause any kind of damages to our sensitive organs such as peripheral nervous system, kidneys, and retinas $[7,8]$.

An actual mechanism reported in a previously studies suggested that the activation of various key pathways at hyperglycemic state which leads to abnormal NF-KB generation, leading to acytokine belonging family mediators' production eg. ICAM-1, VCAM-1, and VEGF expression is occurred due to unwanted reactive oxygen species (ROS) generation such as $\mathrm{H} 2 \mathrm{O} 2$ and superoxide anion, which is directly linked to a vascular injury in retinal structure [9-11]. With this in mind, various antioxidants such as nutraceuticals and natural oxidants have been discovered recently, due to their apparent benefits over diabetes and other complications linked to it [12-15]. Malondialdehyde (MDA), a marker system for peroxidation of lipids present in the erythrocytic membrane of type- 2 diabetic patients. Because MDA act as a marker system, therefore it can be used to diagnose the abnormal developing reactions occurring in diabetic patients and other complication linked to diabetes mellitus (DM). Unwanted Free-radical reactions arise after lipid toxification due to peroxidation of RBC membrane, causing the development of microvascular complications (MVC) like retinopathy in diabetic individuals [16]. This report focused on the relationship between oxidative stress and diabetes mellitus responsible to develop other complication like diabetic retinopathy.

The aim of present study is to establish a correlation between type-2 diabetes mellitus, individuals with/without micro-vascular complications such as neuronal, nephron and retinal related damage to healthy individuals. 


\section{MATERIAL AND METHODS}

This study was conducted in the Department of Endocrinology, Wuhan Fourth Hospital; Puai Hospital, Tongji Medical College, Huazhong University of Science and Technology, Wuhan, Hubei-430034, China.

The total number of one hundred and ten $(n=110)$ individuals were participating in this study, out of which Ten $(n=10)$ were considered as pre-diabetic mellitus (PDM), and rest hundred $(n=100)$ includes: Type2 diabetics (T2D) $(n=50)$ and normoglycaemic $(N G)(n=50)$ group was considered as healthy individuals were treated as subjects of analysis.

\section{Blood Sample Collection}

The waist width of the subjects was measured accurately around the point of the umbilicus by using a non-stretchable plastic tape. From hip portion that is widest portion of the body through which blood is easily drawn out in a measurable amount for analysis.

\section{Experimental procedure}

Ten $\mathrm{mL}$ quantity of fasting venous blood sample is required to collect from $12 \mathrm{hrs}$ fasten individuals. The collected $10 \mathrm{~mL}$ quantity of blood was transferred by sterile syringes to vacutainer tubes to analyze the superoxide dismutase (SOD) activity, malondialdehyde (MDA) and hemoglobulin concentration. Trolox equivalent antioxidant capacity (TEAC) assay, oxygen radical absorption capacity (ORAC) assay were best method to analyze it. EDTA for the ferric reducing ability of plasma (FRAP) assay specifically for $\mathrm{HbA} 1 \mathrm{c}$, fasting plasma glucose, and fasting lipids checking; and rest of the blood serum containing tubes is kept for fasting serum insulin analysis.

Aliquots of the sample was kept at $-80^{\circ} \mathrm{C}$ to analyze the SOD activity. For $\mathrm{HbA} 1 \mathrm{c}$ determination, aliquot of whole blood was stored in deep freezer maintained at $-20^{\circ} \mathrm{C}$. Anticoagulant was added to blood plasma before centrifugation for further analysis of TEAC, ORAC, and MDA, keeping centrifugal tubes at $4^{\circ} \mathrm{C}$ running at $4000 \mathrm{rpm}$ for 10 minutes and kept at $-80^{\circ} \mathrm{C}$ and the rest of the fractionized EDTA-plasma sample was again kept at $-80^{\circ} \mathrm{C}$ for FRAP analysis. Aliquots of plasma and serum were stored at $-20^{\circ} \mathrm{C}$ to determine the sugar content, lipid profile, and insulin in a blood.

\section{Erythrocyte superoxide dismutase (SOD) activities}

The spectrophotometrically method was to determine the activity of Superoxide dismutase (SOD) in erythrocytic cells by using a commercially available kit RANSOD, Randox Lab Ltd, Crumen, UK. Actually, SOD activity is based on the generation of free radicals such as superoxide $\left(\mathrm{O}_{2}{ }^{-}\right)$, produced by xanthine in the presence of xanthine oxidase. The standard experiment of measurement of SOD activity is used by many researcher's [17-19].

\section{Plasma MDA level}

Plasma MDA concentration was determined by using thiobarbituric acid [20] Satoh's method [21,22]. Briefly, $200 \mu \mathrm{L}$ quantity of heparinized blood plasma was taken, then butylated hydroxytoluene (BHT) reagent is added, with the addition of trichloroacetic acid (TCA) to precipitate out plasma proteins. Thio-barbituric acid (TBA) was added to a prepared reaction mixture and incubated at $95^{\circ} \mathrm{C}$ for 45 minutes on a water bath. Chromogenic organic mixture was centrifuged with $\mathrm{n}$-butanol and saturated $\mathrm{NaCl}$ solution. This is colorimetric test which is based on the MDA level corelated with intensity of color. Spectrophotometrically measured at $535 \mathrm{~nm}$ and $572 \mathrm{~nm}$ against the blank.

\section{Glycemic control}

Fasting plasma glucose (FPG) level and $\mathrm{HbA} 1 \mathrm{c}$ levels can be measured by using commercially available enzymatic reagent kits. Enzyme-Linked Immunosorbent Assay (ELISA)-kits used for insulin determinations.

\section{Fasting plasma lipids}

Total cholesterol (TC) and triacylglycerol (TAG) content can be measured by using commercially available enzymatic reagent kits. The level of HDL cholesterol was determined after selective precipitating the VLDL and LDL [23]. The Friedewald formula [24] can be used to calculate the LDL cholesterol content. 
Statistical Analysis- T-2D and PDM groups can be categorized into sub-category, based on good glycemic control with (HbA1c<7\%; (+) GGC) and bad glycemic control with (HbA1c>7\%; (-) GGC) into T2-DM without complication and T2-DM with complication. Basal Metabolic Index (BMl>25 $\mathrm{kgm}-2)$ was used to define obesity [25]. The waist circumference ( $>85 \mathrm{~cm}$ for women and $>90$ for males) and waist to hip ratio i.e. $>0.85$ for females and 0.9 for males) [26] defined the absolute obesity.

Ethics approval and consent to participate- The above study was approved by the Human Ethical Committee (Ethics Committee approval number: WFH/TM/11/19 dated 24 June 2019) of the Wuhan Fourth Hospital; Puai Hospital, Tongji Medical College,Wuhan, Hubei-430034, China and informed consent was obtained from the patients before the study.

\section{RESULTS}

One hundred and ten $(n=110)$ number of individuals were participating in this study. Mostly $70 \%$ males were participating in this study, while female's participation was $30 \%$ only in all of the three groups because at present scenario mostly males are affected by this chronic disease Type-2 diabetes in India. At $p$-value $(P>0.05)$ showed the insignificant difference between groups and gender composition of one group was found to be approximately likewise to that of another group. In the present study, mostly cases of nephropathy with complication (45.67\%) were observed and rest cases were concerning with both nephropathy and retinopathy, it was only $27.67 \%$ cases were found. Some patients were already gaining benefit of antihypertensive therapy. No patients were given insulin injection to both the studied cases of diabetic patients. Table 1 depicts the comparison of selected parameters between the three groups: control, T2DM with complication and T2DM without complication, statistically expressed as mean and standard deviation (mean $\pm S D$ ). An observed study found that older aged (56.19 years) diabetic patients with complication were more prone to be affected significantly (at $p<0.05$ ) than the lesser aged diabetic patients without complications (50.19 yrs). Straightforwardly, it mean solder aged patients were likely chances to suffered from longer duration of illness than those without complication.

Table 1. Comparison between cases: Control and Type-2DM with and without MVC complication.

\begin{tabular}{|c|c|c|c|c|c|}
\hline \multirow[t]{2}{*}{ Parameters } & \multirow{2}{*}{$\begin{array}{l}\begin{array}{l}\text { Control } \\
(n=10)\end{array} \\
\text { Mean } \pm S . D\end{array}$} & \multirow{2}{*}{$\begin{array}{l}\text { T2-DM with } \\
\text { complication } \\
(\mathrm{n}=50)\end{array}$} & \multirow{2}{*}{$\begin{array}{l}\text { T2-DM without } \\
\text { complication } \\
(n=50) \\
\text { Mean } \pm \text { S.D }\end{array}$} & \multirow[t]{2}{*}{ F-value } & \multirow[t]{2}{*}{$p$-value } \\
\hline & & & & & \\
\hline Age (Years) & $44.72 \pm 8.39$ & $56.19 \pm 9.65$ & $50.19 \pm 9.65$ & 11.485 & 0.000 \\
\hline Duration of diabetes & & $10.01 \pm 1.32$ & $5.01 \pm 0.65$ & 111.039 & 0.000 \\
\hline *SOD (U/gHb) & $1079.05 \pm 58.96$ & $660.96 \pm 26.73$ & $856.99 \pm 53.46$ & 829.885 & 0.000 \\
\hline $\operatorname{MDA}(\mu \mathrm{mol} / \mathrm{L})$ & $1.86 \pm 0.11$ & $5.96 \pm 0.38$ & $3.72 \pm 0.15$ & 596.11 & 0.000 \\
\hline $\mathrm{HDL}(\mathrm{mmol} / \mathrm{L})$ & $0.92 \pm 0.23$ & $0.94 \pm 0.27$ & $0.90 \pm 0.21$ & - & 0.077 \\
\hline LDL (mmol/L) & $3.31 \pm 1.00$ & $3.31 \pm 1.00$ & $3.40 \pm 0.70$ & - & 0.121 \\
\hline $\mathrm{TC}(\mathrm{mmol} / \mathrm{L})$ & $4.5 \pm 1.00$ & $4.59 \pm 1.00$ & $4.6 \pm 0.70$ & - & 0.128 \\
\hline $\mathrm{TAG}(\mathrm{mmol} / \mathrm{L})$ & $1.30 \pm 0.62$ & $1.57 \pm 0.78$ & $1.57 \pm 0.85$ & - & 0.030 \\
\hline${ }^{*} \mathrm{FPG}(\mathrm{mmol} / \mathrm{L})$ & $5.1 \pm 0.56$ & $7.89 \pm 2.80$ & $5.77 \pm 1.20$ & - & 0.031 \\
\hline
\end{tabular}

${ }^{*}$ SOD activity was negatively correlated with FPG, HbA1c in T2D, and FPG in PDM patients

Table 2 shows the observed mean value of $\mathrm{HbA} 1 \mathrm{c} \%$ concentration forT-2DM with more complication was $(9.63 \%)$, found to be highest value amongst the examined groups and rest of the group T-2DM without complication and control group with least one i.e. (7.66\% and 5.18\%), compared to complication group. Statistically analysis (at $p<0.05$ level), proves the significant difference between the values of three groups and it clears that there is no glycemic control. In addition, Table.2 depicts the diabetic patients with/without complication groups showing the significantly lower SOD activity than control (at $p<0.05$ level). Table 2 shows the difference between the cases with and without complication based on parameters in terms of 
Mean \pm SD by the using t-test. This study investigated the significant increase in $\mathrm{HbA1c} \%$ concentration in diabetic patients with vascular complications which means glucose control was almost error prone.

Table 2. Comparison between the diabetic cases: with and without MVC complication.

\begin{tabular}{|c|c|c|c|c|}
\hline \multirow[b]{2}{*}{ Parameters } & T-2DM with complication & T-2DM without complication & \multirow[t]{2}{*}{ t-value } & \multirow[t]{2}{*}{ P-value } \\
\hline & Mean $\pm S . D$ & Mean $\pm S . D$ & & \\
\hline Age (Years) & $56.19 \pm 9.65$ & $50.19 \pm 9.65$ & 2.437 & 0.000 \\
\hline Duration of diabetes & $10.01 \pm 1.32$ & $5.01 \pm 0.65$ & 7.239 & 0.000 \\
\hline $\mathrm{HbA1C}(\%)$ & $9.63 \pm 1.37$ & $7.663 \pm 1.29$ & 6.988 & 0.000 \\
\hline SOD (U/gHb) & $660.96 \pm 26.73$ & $856.99 \pm 53.46$ & 17.91 & 0.000 \\
\hline MDA ( $\mu \mathrm{mol} / \mathrm{L})$ & $5.96 \pm 0.38$ & $3.72 \pm 0.15$ & 21.13 & 0.000 \\
\hline
\end{tabular}

Table 3 shows the relationship of highlighted parameters with HbA1c in case of T-2DM without any complication by using correlation coefficient indicated by " $r$ ". There was no significant at ( $p>0.05$ level) correlation between age, duration of diabetes with $\mathrm{HbA1c}$.

Table 3. Correlation between parameters: $\mathrm{HbA1c}(\%)$ and age, duration of diabetes, SOD activity\& MDA concentration in T-2DM without complication.

\begin{tabular}{lcc}
\hline & & HbA1c(\%) \\
\cline { 2 - 3 } Parameters & $r$ & $\mathrm{P}$ \\
\hline Age $(\mathrm{yrs})$ & -0.244 & 0.184 \\
Durationof diabetes $(\mathrm{yrs})$ & 0.116 & 0.512 \\
SOD activity $(\mathrm{U} / \mathrm{gHb})$ & -0.345 & 0.055 \\
${ }^{*} \mathrm{MDA}(\mu \mathrm{mol} / \mathrm{L})$ & 0.31 & 0.119 \\
\hline
\end{tabular}

${ }^{\star}$ Significant correlation was observed at $(p>0.01)$ level.

Table 4 depicts, the correlation coefficient indicated by symbol " $r$ " used to relate the highlighted parameters with $\mathrm{HbA} 1 \mathrm{c} \%$ in T-2DM with MVC complication. Correlation of parameters, namely: patient age, diabetes duration with $\mathrm{HbA} 1 \mathrm{c}$ was not significant statistically at $(\mathrm{p}>0.05)$ level. SOD activity was indirectly correlated but it was insignificant. MDA level was found to be positive correlated to $\mathrm{HbA} 1 \mathrm{c}$ with highly significant value at $(p<0.01)$ level. In addition, multiple regression analysis was performed on diabetic patients with complication, it was clearly shown malondialdehyde (MDA) level is independently related to $\mathrm{HbA} 1 \mathrm{c} \%$ $\left(R^{2}=0.340\right.$ at $\mathrm{P}<0.05$ level) concentration.

Table 4. Correlation between parameters: $\mathrm{HbA} 1 \mathrm{c}(\%)$ and age, diabetes duration, SOD activity \& MDA concentration in T-2DM with complication.

\begin{tabular}{lcc}
\hline & \multicolumn{2}{c}{ HbA1c (\%) } \\
\cline { 2 - 3 } Parameters & $\mathbf{r}$ & $\mathbf{P}$ \\
\hline Age $(\mathrm{yrs})$ & 0.055 & 0.763 \\
Duration of diabetes(yrs) & -0.016 & 0.399 \\
SOD $(\mathrm{U} / \mathrm{gHb})$ & -0.388 & 0.449 \\
${ }^{*} \mathrm{MDA}(\mu \mathrm{mol} / \mathrm{L})$ & 0.547 & 0.002 \\
FPG (SOD activity) & -0.20 & 0.172 \\
FPG (MDA level) & 0.20 & 0.11
\end{tabular}

${ }^{*}$ Significant correlation was observed at (0.01) level. 


\section{DISCUSSION}

Present study results showed the lowest activity of SOD was found in case of diabetic patients with complication; automatically enhance the lipid peroxides (malondialdehyde) level, compared to deprive of MVC complication or control group. A significant decrement in SOD activity observed in case of diabetics with MVC indicating lack of antioxidants, which directly initiates the reactive oxygen species generation [3,25]. The plasma MDA level was also affected and its level found to be higher in case of diabetic with complication, indicating more reactive species generation to reduce the lipids due to oxidative stress [26,23]. It was observed in this study that parameters such as blood glucose, $\mathrm{HbA} 1 \mathrm{c} \%$, and MDA levels were increased significantly in complication cases compared to without complications cases. These factors are likely to be related with each other, because the lowest level of metformin changes the situation to a poor glycemic control leading to more oxidative stress, and diverting towards more prone to developing the late diabetic complications [21].

In this current study, no significant relation was obtained while studying the parameters: high-density lipoprotein (HDL), low density lipoprotein (LDL), Total cholesterol (TC) and Triacylglycerol (TAG) level in T2DM with/ without complication compared to Control group or no correlation was found with MDA level. Previous studies reported the MDA level changes significantly when the oxidation of unsaturated fatty acids occur similar changes found in triglyceride structure $[27,28]$. The $\mathrm{HbA} 1 \mathrm{c} \%$ shows the negatively correlated to superoxide dismutase (SOD) but positively correlated to malondialdehyde (MDA) but it was not significant. Other authors also reported same thing as our study shows the positive correlation between glycemic control parameters and MDA in DM patients [17,18,29,30] due to excessive accumulation of lipid peroxidation products. Our study revealed that SOD activity was decreased as in case of T-2DM with complication than without complication and control group due to auto-oxidation of glucose and more $\mathrm{H} 2 \mathrm{O} 2$ production, but it was reverse in other reported studies due to increased protein glycation, which leads to reduced vascular concentrations of SOD-3 in relation to intracellular SOD-1 activity is also decreased, resulting into an increased superoxide production $[22,31,32]$.

\section{CONCLUSION}

By analyzing the whole results carefully in this study, it can be concluded that oxidative stress was more dominant reasons in case of type-2 diabetic patients in those who have poor glycemic control with more complications. The basic reason behind these complications like diabetes retinopathy (DR) was declined in SOD activity and enhances the MDA level by excessive generation of $\mathrm{H}_{2} \mathrm{O}_{2}$ and $\mathrm{O}_{2}^{-}$species due to autooxidation of glucose, lipid peroxidation and protein glycation present in hyperglycemic state. From this study, at hyperglycemia condition also induces the reactive species generation like superoxides and hydrogen peroxides, which automatically starts to excessive use of endogenous antioxidants already available in plasma that is prominently observed in case of T-2 DM with complication as compared T-2 DM without complication and normoglycemia/control group.

However, there is a presence of lots of controversial data on the antioxidant enzymes related to diabetes mellitus (DM) therefore, there is the need for long-term fruitful studies in order to analyze a large set of the considered parameters carefully and highlight the actual mechanisms involved in the complex pathophysiology of T2-DM.

Funding:This study was supported by Wuhan health and family planning commission (No: WZ18Q18).

Conflicts of Interest:Authors declare that they have no conflict of interest relevant to the content of this study.

\section{REFERENCES}

1. Shaw JE, Sicree RA, Zimmet PZ. Global estimates of the prevalence of diabetes for 2010 and 2030. Diabetes Res Clin Pract. 2010; 87(1): 4-14.

2. Yuan $\mathrm{T}$, Yang $\mathrm{T}$, Chen $\mathrm{H}$, et al. New insights in to oxidative stress and inflammation during diabetes mellitus-accelerated atherosclerosis. Redox Biol. 2019;20:247-60.

3. Zheng Y, He M, Congdon N. The worldwide epidemic of diabetic retinopathy. Indian J Ophthalmol. 60(5): 2012; 428-31.

4. Distefano LN, Garcia-Arumi J, Martinez-Castillo V, Boixadera A. Combination of anti-VEGF and laser photocoagulation for diabetic macular edema: a review. J Ophthalmol. 2017; 1-7. 
5. Claramunt J. Diabeticretinopathy. Rev. Méd. Clín. Las Condes. 2009; 20(5): pp. 670-679.

6. Koopman WJH, Nijtmans LGJ, Dieteren CEJ, et al. Mammalian mitochondrial complex I: biogenesis, regulation, and reactive oxygen species generation. Antioxidants \& Redox Signaling. 2010; 12(12): 14311470.

7. Park S, Kang HJ, Jeon JH, Kim MJ, Lee IK. Recent advances in the pathogenesis of microvascular complications in diabetes. Arch. Pharm. Res. 2019; 42(3): 252-62.

8. Barrett EJ, Liu Z, Khamaisietal M. Diabetic microvascular disease: an Endocrine Society scientific statement. J. Clin. Endocrinol. Metab. 2017; 102(12): 4343-4410.

9. Shaw JE, Sicree RA, Zimmet PZ. Global estimates of the prevalence of diabetes for 2010 and 2030. Diabetes Res. Clin. Pract. 2010; 87(1): 4-14.

10. Aghadavod E, Khodadadi S, Baradaran A, Nasri P, et al. Role of oxidative stress and inflammatory factors in diabetic kidney disease. Iran. J. Kidney Dis. 2016; 10(6): 337-343.

11. Jha JC, Banal C, Chow BSM, et al. Diabetes and kidney disease: role of oxidative stress. Antioxidants and Redox Signaling, 2016; 25(12): 657-684.

12. Sifuentes-Franco S, Pacheco-Moisés FP, RodríguezCarrizalez AD, Miranda-Díaz AG. The role of oxidative stress, mitochondrial function, and autophagy in diabetic polyneuropathy. J. Diabetes Res., 2017; 2017: 1673081. doi: 10.1155/2017/1673081.

13. Weng L, Zhang F, Wang R, Ma W, Song Y. A review on protective role of geniste in against oxidative stress in diabetes and related complications. Chem. Biol. Interact, 2019; 310.

14. Bao L, Li J, Zhaetal, D. Chlorogenic acid prevents diabetic nephropathy by inhibiting oxidative stress and inflammation through modulation of the Nrf2/HO-1 and NF-kB pathways," Int. Immunopharmacol. 2018; (54): 245-253.

15. Rossino MG, Casini G. Nutraceuticals for the treatment of diabetic retinopathy," Nutrients, 2019; 11(4):771.

16. Laddha AP, Kulkarni YA. Tannins and vascular complications of diabetes: an update. Phytomedicine, 2019;(56):229-45.

17. Singh R, Hijam D, Dubey A, Devi NO, Jamir S, Longkumer C, et al. Study of Oxidative Stress Status In Type 2 Diabetic Patients. Int. J. Contemp. Med. Res. 2015;2(1):20-6.

18. Karasu C. Glycoxidative stress and cardiovascular complications in experimentally-induced diabetes: effects of antioxidant treatment. Open Cardiovasc Med. J. 2010; 26(4): 240-256.

19. Bouchoucha M, Uzzan B, Cohen R. Metformin and digestive disorders. Diabetes Metab. 2011; 37(2): 906

20. Sekhar RV, McKay SV, Patel, SG, Guthikonda, AP, Reddy VT. Glutathione synthesis is diminished in patients with uncontrolled diabetes and restored by dietary supplementation with cysteine and glycine. Diabetes Care. 2011;34(1):162-67

21. Migdal C, Serres M. Reactive oxygen species and oxidative stress. Med Sci (Paris) 2011; 27(4):405-12.

22. Sasaki S, Inoguchi T. The role of oxidative stress in the pathogenesis of diabetic vascular complications. Diabetes Metab. J. 2012; 36(4): 255-61

23. Giacco F, Brownlee M. Oxidative stress and diabetic complications. Circulation Research. 2010; 107(9):1058 -70.

24. Ovadia H, Haim Y, Nov O, Almog O, Kovsan J, Bashan N, Beenhar M, Rudich A. Increased adipocyteS-nitrosylation targets anti-lipolytic action of insulin relevance to adipose tissue dysfunction in obesity. J. Biol. Chem. 2011; 286(35): 30433-43.

25. Filla LA, Edwards JL. Metabolomics in diabetic complications. Mol Biosyst. 2016;12(4):1090-105.

26. The World Health Report 2008- primary Health Care (Now More Than Ever, 2008.

27. Murthy G, Das T. Diabetic care initiatives to prevent blindness from diabetic retinopathy in India. Indian J Ophthalmol. 2016 Jan;64(1):50-4.

28. Antunes MV, Wagner SC, Camargo JL, Linden R. Standardization of method for determining glycosylated hemoglobin $(\mathrm{Hb} \mathrm{A} 1 \mathrm{c})$ by cation exchange high performance liquid chromatography. Braz $\mathrm{J}$ Pharm. 2009;45(4):650-7

29. Khan S, Naveed AK, Shabbir F, Rajput T A, Yousaf MJ. Oxidative Stress in Patients with Type 2 Diabetes Mellitus. J. Rawalpindi Med. Coll. 2014;18(1):29-31.

30. Dries SS, Soares BS, Verza SG, Ziulkoski AL, Linden R, Andrade FM, Perassolo MS. Oxidative stress in patients with type 2 diabetes mellitus treated with metformin. Sci. Med. 2017;27(2):1-8. 
31. Lang VB, Marković BB. Prevalence of comorbidity in primary care patients with type 2 diabetes and its association with elevated HbA1c: A cross-sectional study in Croatia. Scand J Prim Health. 2016; 34(1): 66-72.

32. Matough FA, Budin SB, Hamid ZA, Alwahaibi N, Mohamed J. The Role of Oxidative Stress and Antioxidants in Diabetic Complications. Sultan Qaboos Univ Med J. 2012; 12(1): 5-18.

(C) (7) \&) $\begin{aligned} & @ 2021 \text { by the authors. Submitted for possible open access publication under the } \\ & \text { terms and conditions of the Creative Commons Attribution (CC BY NC) license } \\ & \text { (https://creativecommons.org/licenses/by-nc/4.0/). }\end{aligned}$ 